\title{
Solid-liquid phase transition and heat engine in an asymptotically flat Schwarzschild black hole via the Rényi extended phase space approach
}

\author{
Chatchai Promsiri®,$^{1,2, *}$ Ekapong Hirunsirisawat $\odot,{ }^{2,3, \dagger}$ and Watchara Liewrian $\oplus^{1,2,4, \hbar}$ \\ ${ }^{1}$ Department of Physics, Faculty of Science, King Mongkut's University of Technology Thonburi, \\ Pracha Uthit Road, Bangkok, 10140, Thailand \\ ${ }^{2}$ Theoretical and Computational Physics (TCP); Theoretical and Computational Science Center (TaCS), \\ Faculty of Science, King Mongkut's University of Technology Thonburi, \\ Pracha Uthit Road, Bangkok, 10140, Thailand \\ ${ }^{3}$ Learning Institute, King Mongkut's University of Technology Thonburi, \\ Pracha Uthit Road, Bangkok, 10140, Thailand \\ ${ }^{4}$ Thailand Center of Excellence in Physics, Ministry of Higher Education, Science, \\ Research and Innovation, 328 Si Ayutthaya Road, Bangkok, 10400, Thailand
}

(Received 18 June 2021; accepted 20 July 2021; published 2 September 2021)

\begin{abstract}
Recently, it has been found that, with the Rényi statistics, the asymptotically flat Schwarzschild black hole can be in thermal equilibrium with infinite heat reservoir at a fixed temperature when its event horizon radius is larger than the characteristic length scale $L_{\lambda}=1 / \sqrt{\pi \lambda}$, where $\lambda$ is the nonextensivity parameter. In the Rényi extended phase space with the $P d V$ work term, an off shell free energy in the canonical ensemble with the thermodynamic volume as an order parameter is considered to identify a first-order Hawking-Page (HP) phase transition as a solid-liquid phase transition. It has the latent heat of fusion from solid (corresponding to thermal radiation) to liquid (corresponding to black hole) in the form of $\sim 1 / \sqrt{\lambda}$; this is evident in the absence of the HP phase transition in the case of an asymptotically flat Schwarzschild black hole from the Gibbs-Boltzmann statistics $(\lambda=0)$. Moreover, we investigate the generalized second law of black hole thermodynamics (GSL) in Rényi statistics by considering the black hole as a working substance in a heat engine. Interestingly, an efficiency $\eta$ of the black hole in a Carnot cycle takes the form $\eta_{c}=1-T_{\mathrm{C}} / T_{\mathrm{H}}$. This confirms the validity of the GSL in the Rényi extended phase space.
\end{abstract}

DOI: 10.1103/PhysRevD.104.064004

\section{INTRODUCTION}

The notion of a black hole having the nature of a thermal object originated from the surprising mathematical parallels between the laws of black hole mechanics and of thermodynamics [1]. Since then, the geometrical properties of a black hole's event horizon has been found to be linked with its thermodynamic properties. Bekenstein was the first to postulate that the surface gravity and the area of a black hole's event horizon could be regarded as the temperature and the entropy of black hole, respectively [2]. While the geometrical considerations through general relativity cannot give a black hole any thermal concept, the Hawking's

*chatchaipromsiri@gmail.com

†ekapong.hir@mail.kmutt.ac.th

watchara.liewrian@mail.kmutt.ac.th

Published by the American Physical Society under the terms of the Creative Commons Attribution 4.0 International license. Further distribution of this work must maintain attribution to the author(s) and the published article's title, journal citation, and DOI. Funded by SCOAP. consideration on the quantum effect around the event horizon predicts the emission of thermal radiations at a certain temperature to an observer at infinity [3]. The need of taking into account the quantum properties in black holes may imply that the black hole thermodynamics may be related in some ways with the quantum theory of gravity.

Recently, there have been some concerns as to whether the conventional thermodynamics, based on the GibbsBoltzmann (GB) statistics, is valid in deriving the thermodynamic properties of an extreme situation like a black hole. It is essential to elaborate here the important issues of black hole thermodynamics from the GB statistical approach, which could not be resolved satisfactorily. They are as follows:

(i) The GB statistics is appropriate to describe only a weakly interacting system whose the entropy is extensive. Nevertheless, the black hole system has long-range gravitational interactions and, according to some clues, may have nonlocal quantum correlations among its constituents, such that it should not be an extensive system. This is evident through the area law of its entropy [4-6]. Therefore, a black hole 
is a nonextensive system, which cannot be described well using the thermodynamics based on the GB statistics.

(ii) Based on the GB statistics, a black hole surrounded by an infinite bath of thermal radiation in an asymptotically flat spacetime is unstable due to the presence of its negative heat capacity. This implies that the canonical ensemble in the GB statistics cannot be applied in the case of a nonextensive long-range interaction black hole system in asymptotically flat spacetime at a fixed temperature [7]; for a good review, see [8].

(iii) Basically, the volume within a black hole's event horizon can expand when it absorbs some energy, whereas it can shrink as it emits the Hawking's radiation. One may wonder whether the thermodynamic system corresponding to the black hole can perform the mechanical work in a similar way as occurs in a gas system in the GB thermodynamics. Unfortunately, the notion of thermodynamic pressure and volume are absent in standard black holes physics. Therefore, the first law of black hole thermodynamics does not include the mechanical work $P d V$ term to describe the change of internal energy in thermodynamic processes.

To address these issues, we may need to use an alternative choice of the generalized entropy function. Recently, the Tsallis [9] and Rényi [10] entropies, which are based on the power law probability distribution, has been used to study strongly interacting phenomena in particle physics [11-13], kinetic theory [14-16], and complex systems [17]. A comprehensive description of generalized entropies and its applications has been found in [18]. In black hole physics, its thermodynamic nature was first explored via the nonextensive extropy by Tsallis and Cirto [19]. The thermodynamic stability and phase structure of the Schwarzschild, Kerr, and Reissner-Nordström (RN) black holes in an asymptotically flat spacetime were investigated via Rényi statistics in [20-22]. These two nonextensive entropies have the parameter $\lambda$, which is the so-called nonextensive parameter. The deviation of its value from zero quantifies how much the system's behaviors deviate from the extensive GB statistics, corresponding to $\lambda=0$.

Studying with the Rényi statistics, the black holes in asymptotically flat spacetime can be in stable thermodynamic equilibrium with thermal bath at a fixed temperature as $\lambda$ above zero. Hence, the canonical ensemble of this system exists in a case of nonextensive Rényi statistics. The role of the nonextensive parameter $\lambda$ to stabilize the black holes is in a similar fashion as the gravitational potential with negative cosmological constant $\Lambda$ in anti-de Sitter (AdS) space. Moreover, the nonextensive effect can stabilize the Schwarzschild black hole in a de Sitter spacetime of the positive cosmological constant as well $[23,24]$.
As mentioned above in (iii), the notion of thermodynamic pressure and the volume of black holes are absent such that some thermodynamic behaviors of black holes are not allowed to mathematically match with those in the thermodynamics of a conventional matter. For instance, even though it has been found that the van der Waals ( $\mathrm{vdW}$ )-like phase transition exists in the $q-\phi$ plane of the AdS Reissner-Nordström black hole in a canonical ensemble, we cannot exhibit this phase transition in the $P-V$ plane [25-28]. A more complete analogy between the conventional matter and a black hole has been established by introducing a negative cosmological constant $\Lambda$ as a thermodynamic variable, namely the thermodynamic pressure $P=-\frac{\Lambda}{8 \pi}$. Its conjugate is the thermodynamic volume $V=\frac{4}{3} \pi r_{h}^{3}$, where $r_{h}$ is an event horizon radius. In this approach, the mass turns out to be an enthalpy that can be written as $M=H=U+P V[29,30]$. This framework is known as black hole thermodynamics in the extended phase space or black hole chemistry (for review, see [31-33] and references therein).

Without the negative cosmological constant, the asymptotically flat black hole for example has the vanishing pressure, and hence the notion of thermodynamic pressure and volume is absent. Fortunately, the authors in [22] have proposed an idea that the nonextensive parameter $\lambda$ can be regarded as a thermodynamic variable. As a result, $\lambda$ is allowed to play a role of the thermodynamic pressure. In this way, we identified the thermodynamic pressure $P=\frac{3 \lambda}{32}$ and its conjugate variable as the thermodynamic volume $V=\frac{4}{3} \pi r_{h}^{3}$. In addition, the consistent Smarr formula from the scaling argument has revealed that the black hole mass $M$ should be interpreted as an enthalpy instead of the internal energy of the black hole. This is in the same way as the extended phase space in AdS, as mentioned above. This framework is called the Rényi extended phase space approach or may be dubbed the Rényi black hole chemistry.

Intriguingly, it has been found in [22] that there exists a vdW-like phase transition of the flat RN black hole in this framework. One may wonder whether there exists some interesting phase transitions in the asymptotically flat Schwarzschild black hole (Sch-flat) from the Rényi extended phase space approach. Hence, applying this in a variety of cases is necessary for testing its validity and exploring some interesting aspects of this new approach. To achieve this, we consider in this paper the Gibbs free energy, using this approach to investigate a first-order radiation, large black hole phase transition in the asymptotically flat background.

Moreover, it is interesting to investigate whether the thermodynamic process from this setup obeys the generalized second law of thermodynamics (GSL), which states that the sum of black hole entropy and that of the environment outside the horizon never decreases [34]. The GSL is stronger than a classical area theorem [35] 
because it is also valid when the Hawking radiation is taken into account. A black hole emits particles via the process of Hawking radiation. Consequently, the area of its event horizon becomes smaller, implying a decrease in the black hole's entropy. This semiclassical process has therefore violated the classical area theorem and also the second law of thermodynamics. However, the GSL is still valid despite the decrease of horizon area; the increase in the entropy of the environment occupied by Hawking radiations emitted from the black hole overcomes the decrease of Bekenstein-Hawking entropy such that the overall entropy keeps growing up.

There have been several attempts to test the validity of the GSL; for a review, see [36,37]. In the context of the extended phase space approach, the holographic heat engine in the $P-V$ plane through considering the AdS black hole as a working substance was proposed by Johnson and Chakraborty [38-40]. Several holographic heat engines were studied later in [41-45]. Inspired by Johnson and Chakraborty's work, the thermal efficiency of a black hole heat engine will be derived in the present paper to investigate the GSL validity from the macroscopic point of view, instead of the notion of information theoretic and statistical arguments. From the Carnot statement that "no heat engine, operating between the same high and low temperatures, can be more efficient than a Carnot engine" [46], the upper bound of the efficiency coefficient is in the form $\eta_{c}=1-\frac{T_{\mathrm{C}}}{T_{\mathrm{H}}}$. In other words, the existence of a heat engine with $\eta>\eta_{c}$ violates the second law of thermodynamics. Applying the Rényi extended phase space in the Sch-flat can give the corresponding reversible thermodynamic processes in a heat engine, which can be defined as a closed path in the $P-V$ diagram. The presence of the $P d V$ term allows us to consider the mechanical work from the expansion and contraction of a black hole, in the same way as conventional matter. Thus, apart from exploring the phase transition, we consider in the present work a Carnot cycle in the $P-V$ diagram and determine a thermal efficiency coefficient of the black hole heat engine. This is an additional validity test of the Rényi extended phase space approach in black hole thermodynamics to ensure that the GSL is satisfied.

This paper is organized as follows. We begin with the review of nonextensive statistics, namely the Tsallis and Rényi statistics and the deep reasons why we need it in the consideration of black hole thermodynamics in Sec. II. Next, the GB and Rényi thermodynamics of Sch-flat are reviewed in Sec. III. The thermodynamic behaviors of black holes from the Rényi entropy are also discussed in this section. Moreover, the formulation of black hole thermodynamics of Sch-flat in the Rényi extended phase space is derived using the generalized Smarr formula in Sec. IV. With this approach, the Hawking-Page phase transition of the Sch-flat black hole corresponding to the solid-liquid phase transition in conventional matter and its latent heat are discussed in Sec. V. Importantly, the cyclic thermodynamic process of the Sch-flat from the Rényi statistics, namely the black hole heat engine, is discussed in Sec. VI. We end with the conclusion in Sec. VII.

\section{TSALLIS AND RÉNYI STATISTICS}

A nonextensive generalization of GB entropy was proposed by Tsallis [9]. For a system of $W$ discrete states, the Tsallis entropy can be written in the form

$$
S_{\mathrm{T}}=\frac{1}{1-q}\left(\sum_{i=1}^{W} p_{i}^{q}-1\right),
$$

where $p_{i}$ is the probability associated with a state $i$ and $q \in \mathbb{R}$ is a real parameter. Defining a nonextensivity parameter $\lambda \equiv 1-q$, the above formula becomes the GB entropy when $\lambda \rightarrow 0$. It is noteworthy here to indicate that the GB entropy is associated with the extensive system. Considering a combined system $\mathrm{AB}$ consisting of the subsystems $\mathrm{A}$ and $\mathrm{B}$, its entropy is extensive when it can be written in the form $S\left(W_{\mathrm{AB}}\right)=S\left(W_{\mathrm{A}}\right)+S\left(W_{\mathrm{B}}\right)$, where $W_{\mathrm{A}}, W_{\mathrm{B}}$, and $W_{\mathrm{AB}}$ are the number of states of the systems $\mathrm{A}, \mathrm{B}$, and $\mathrm{AB}$, respectively. Note that $W_{\mathrm{AB}}=$ $W_{\mathrm{A}} W_{\mathrm{B}}$ when $\mathrm{A}$ and $\mathrm{B}$ are independent. However, the formula of entropy in (1) is in the nonextensive form, which is relaxed to a weaker nonadditive composition rule [47]. The Tsallis entropy of a combined system can be written as

$S_{\mathrm{T}}\left(W_{\mathrm{AB}}\right)=S_{\mathrm{T}}\left(W_{\mathrm{A}}\right)+S_{\mathrm{T}}\left(W_{\mathrm{B}}\right)+\lambda S_{\mathrm{T}}\left(W_{\mathrm{A}}\right) S_{\mathrm{T}}\left(W_{\mathrm{B}}\right)$.

Note that this composition rule becomes additive when $\lambda=0$, corresponding to that of the GB entropy. However, the composition rule of the Tsallis entropy turns out to be in the additive form using an appropriate transformation. We will come back to this point later.

Here, let us introduce another generalized entropy with $\lambda$ parameter, the Rényi entropy [10], which is defined as

$$
S_{\mathrm{R}}=\frac{1}{\lambda} \ln \sum_{i=1}^{W} p_{i}^{1-\lambda}
$$

We remark that the nonextensivity parameter $\lambda$ of the Rényi entropy has to be not more than 1, otherwise the entropy function becomes not well defined due to its convexity. This issue can be illustrated more through considering a biased coin with the probability of landing on heads and tails is $p$ and $1-p$, respectively. Using (3), the Rényi entropy of this system can be written in the form

$$
S_{\mathrm{R}}(p)=\frac{1}{\lambda} \ln \left[p^{1-\lambda}+(1-p)^{1-\lambda}\right]
$$

As shown in Fig. 1, the entropy function of $\lambda>1$ (orange, solid) is very large when $p \rightarrow 0$ or 1 . This is not well 


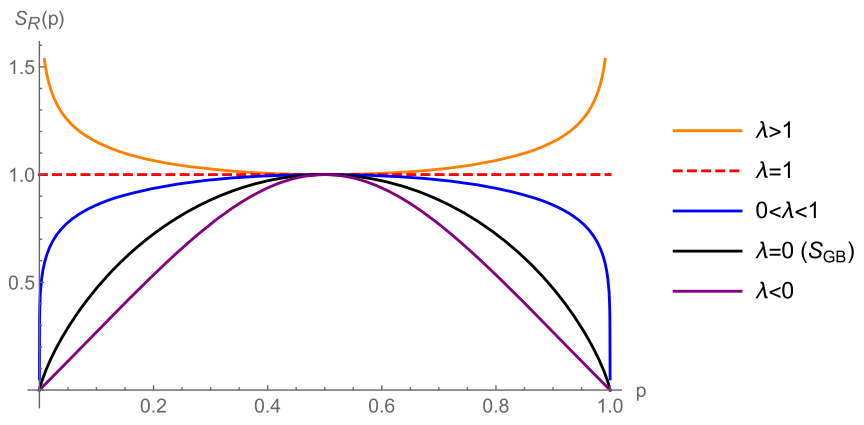

FIG. 1. The Rényi entropy $S_{\mathrm{R}}$ of the system of a biased coin with a random variable of two possible outcomes, namely heads or tails, is plotted as a function of the probability of landing on heads $p$ at different values of $\lambda$. Note that, at all values of $\lambda \leq 1$, the Rényi entropy becomes 1 when $p=0.5$. Namely, a fair coin corresponds to the case which has the maximum value of surprisal.

behaved, since at $p \rightarrow 0(p \rightarrow 1)$, corresponding to the high probability of the coin landing on tails (heads), the outcome should have a very small value of entropy. Namely, the entropy of a biased coin should be maximum at $p=0.5$ (fair coin) and vanishes at $p=0$ and 1 , as can be seen in the plots of the entropy function with $\lambda=1$ (red, dashed), $0<\lambda<1$ (blue, solid), $\lambda=0$ (black, solid), and $\lambda<0$ (purple, solid). In other words, a well-defined entropy function needs to satisfy the concavity property of entropy $[17,18]$. In the present work on black hole thermodynamics with Rényi statistics, the parameter $\lambda$ in range $0<\lambda<1$ is of our interest due to its good thermodynamic behavior as shown in [22].

Considering (1) and (3), it can be shown that the Rényi entropy $S_{\mathrm{R}}$ can be written in terms of the Tsallis entropy $S_{\mathrm{T}}$ as

$$
S_{\mathrm{R}}=\frac{1}{\lambda} \ln \left(1+\lambda S_{\mathrm{T}}\right)
$$

From this transformation, the Rényi entropy of the combined system $A B$ should be written in the form

$$
S_{\mathrm{R}}\left(W_{\mathrm{AB}}\right)=\frac{1}{\lambda} \ln \left(1+\lambda S_{\mathrm{T}}\left(W_{\mathrm{AB}}\right)\right) .
$$

By substituting the nonadditive composition rule (2) for $S_{\mathrm{T}}$, we have

$$
\begin{aligned}
S_{\mathrm{R}}\left(W_{\mathrm{AB}}\right)= & \frac{1}{\lambda} \ln \left[1+\lambda\left(S_{\mathrm{T}}\left(W_{\mathrm{A}}\right)+S_{\mathrm{T}}\left(W_{\mathrm{B}}\right)\right.\right. \\
& \left.\left.+\lambda S_{\mathrm{T}}\left(W_{\mathrm{A}}\right) S_{\mathrm{T}}\left(W_{\mathrm{B}}\right)\right)\right] \\
= & \frac{1}{\lambda} \ln \left[\left(1+\lambda S_{\mathrm{T}}\left(W_{\mathrm{A}}\right)\right)\left(1+\lambda S_{\mathrm{T}}\left(W_{\mathrm{B}}\right)\right)\right] \\
= & \frac{1}{\lambda} \ln \left(1+\lambda S_{\mathrm{T}}\left(W_{\mathrm{A}}\right)\right)+\frac{1}{\lambda} \ln \left(1+\lambda S_{\mathrm{T}}\left(W_{\mathrm{B}}\right)\right) \\
= & S_{\mathrm{R}}\left(W_{\mathrm{A}}\right)+S_{\mathrm{R}}\left(W_{\mathrm{B}}\right) .
\end{aligned}
$$

Therefore, the transformed entropy function, i.e., the Rényi entropy, comes to have additivity, although it is obtained from the nonadditive Tsallis entropy [48].

In thermal equilibrium of an isolated system with two subsystems, the total entropy has the maximum value, satisfying the condition $d S=d S_{\mathrm{A}}+d S_{\mathrm{B}}=0$. According to the zeroth law of thermodynamics, the empirical temperature of these two subsystems are equal, and thus it can be defined via the relation $T^{-1}=\frac{\partial S_{\mathrm{A}}}{\partial E_{\mathrm{A}}}=\frac{\partial S_{\mathrm{B}}}{\partial E_{\mathrm{B}}}$. This indicates that the lack of additive form in nonextensive entropy tends to obscure the notion of thermal equilibrium and empirical temperature. Since the Rényi entropy is additive, we therefore have the entropy compatible with the zeroth law of thermodynamics for describing a nonextensive system. Hence, the empirical temperature function can be obtained from the usual relation

$$
\frac{1}{T_{\mathrm{R}}}=\frac{\partial S_{\mathrm{R}}}{\partial E}
$$

Recently, it has been argued that the BekensteinHawking entropy $S_{\mathrm{BH}}$ of a black hole can be associated with the nonextensive Tsallis entropy [19]. As mentioned above, concerning the zeroth law compatibility, it is thus appropriate to work on black hole thermodynamics using the Rényi entropy as a function the Bekenstein-Hawking entropy in the form

$$
S_{\mathrm{R}}=\frac{1}{\lambda} \ln \left(1+\lambda S_{\mathrm{BH}}\right) .
$$

Using (8) and (9), we can write the Rényi temperature $T_{R}$ in terms of the Hawking temperature $T_{\mathrm{H}}$

$$
T_{\mathrm{R}}=T_{\mathrm{H}}\left(1+\lambda S_{\mathrm{BH}}\right) \text {. }
$$

The thermodynamic properties and phase transitions in several kinds of black hole solutions with the Rényi entropy have been studied recently in [20-23].

\section{RÉNYI STATISTICS AND THERMODYNAMICS OF BLACK HOLES}

In this section, we review the standard GB and Rényi thermodynamics of a Schwarzschild black hole in asymptotically flat spacetime. The line element of spherically symmetric black holes can be written in the form

$$
d s^{2}=-f(r) d t^{2}+\frac{d r^{2}}{f(r)}+r^{2}\left(d \theta^{2}+\sin ^{2} \theta d \phi^{2}\right) .
$$

For the Schwarzschild black hole solution, we have

$$
f(r)=1-\frac{2 M}{r}
$$



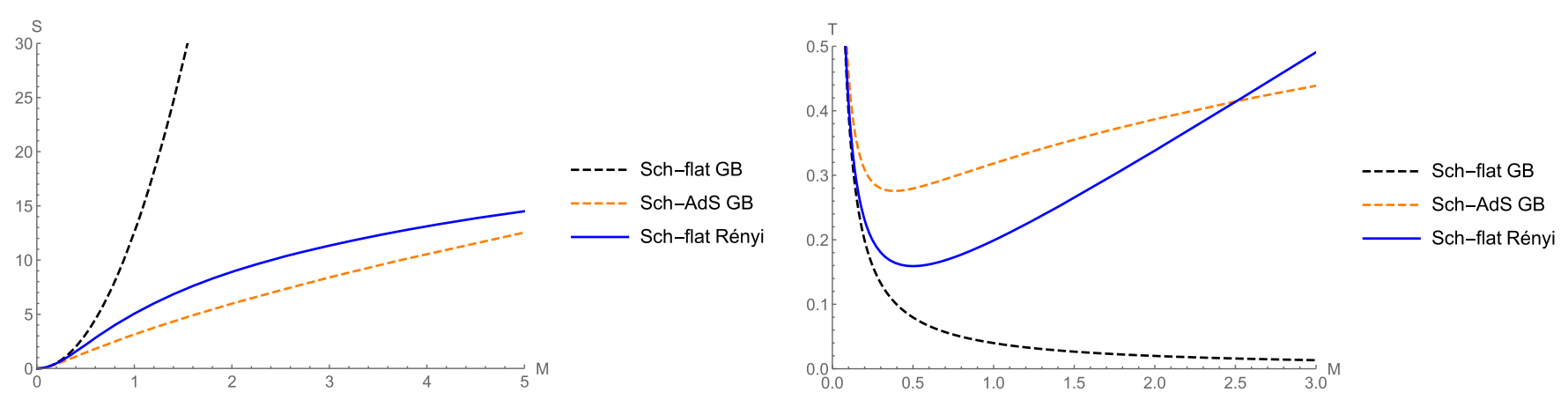

FIG. 2. Left: entropy versus the mass (energy) of a black hole is plotted for the Sch-flat (dashed black) and the Sch-AdS (dashed orange) cases from the GB statistics, compared with one for the case of the Sch-flat from the Rényi statistics $L_{\lambda}=1$ (solid blue). Right: the figure shows the temperature versus the mass (energy) relation of the black hole. Unlike the Sch-flat in GB statistics case, the thermal behavior of black hole in Rényi framework has an interesting similarity to the AdS black hole. In the Rényi approach, this result reveals that the black hole behaves like it is placed in the background with nonzero energy density, in the same way as occurs in the AdS black hole system.

where the parameter $M$ describes the mass of black hole. At the black hole horizon, we have $f\left(r_{h}\right)=0$, where $r_{h}$ is the location of its event horizon. Consequently, the thermodynamic quantities can be written in the black hole's event horizon $r_{h}$ as

$$
\begin{gathered}
M=\frac{r_{h}}{2}, \\
T_{\mathrm{H}}=\frac{f^{\prime}\left(r_{h}\right)}{4 \pi}=\frac{1}{4 \pi r_{h}}, \\
S_{\mathrm{BH}}=\frac{\mathcal{A}}{4}=\pi r_{h}^{2}, \\
C=T_{\mathrm{H}}\left(\frac{\partial S_{\mathrm{BH}}}{\partial T_{\mathrm{H}}}\right)=-2 \pi r_{h}^{2},
\end{gathered}
$$

where $T_{\mathrm{H}}$ is the Hawking temperature, and $S_{\mathrm{BH}}$ is the Bekenstein-Hawking entropy which depends on the surface area of the event horizon $\mathcal{A}$. Note that, with the GB statistics, the heat capacity $C$ has a negative value, implying that the asymptotically flat Schwarzschild black hole is thermodynamically unstable.

From (9) and (10), the Rényi entropy and its corresponding Hawking temperature of the asymptotically flat Schwarzschild black hole takes the form

$$
S_{\mathrm{R}}=\frac{1}{\lambda} \ln \left(1+\lambda \pi r_{h}^{2}\right)=\pi L_{\lambda}^{2} \ln \left(1+\frac{r_{h}^{2}}{L_{\lambda}^{2}}\right),
$$

and

$$
T_{\mathrm{R}}=\frac{1}{4 \pi r_{h}}\left(1+\lambda \pi r_{h}^{2}\right)=\frac{1}{4 \pi r_{h}}\left(1+\frac{r_{h}^{2}}{L_{\lambda}^{2}}\right),
$$

where we have defined the characteristic length $L_{\lambda}=1 / \sqrt{\pi \lambda}$. Obviously, this nonextensivity length scale emerges as a result from the Rényi statistics; $L_{\lambda}$ becomes infinite when we back to the GB statistics, as $\lambda$ approaches zero. For comparison, we plot the entropy and temperature versus the mass (energy) of black holes for the Sch-flat and the Sch-AdS in the standard approach and those for the Sch-flat in the alternative Rényi statistics, as shown in Fig. 2. Note that $\lambda$ is a dimensionless parameter, and we perform the dimensional analysis of (17) to obtain a physical nonextensivity length scale. The physical length scale is given by $L_{\lambda}=l_{P} / \sqrt{\lambda \pi}$, where $l_{P}$ is the Planck length. Remarkably, this length scale should be larger than $l_{P}$, thus we have

$$
L_{\lambda}=\frac{l_{P}}{\sqrt{\lambda \pi}}>l_{P}
$$

which provides the upper bound of the nonextensive parameter due to the quantum gravity limit, i.e.,

$$
\lambda<\frac{1}{\pi}
$$

Thus, it makes sense to consider black hole thermodynamics from the Rényi statistics with the value of $\lambda$ large enough to see an effect of the deviation from the GB thermodynamics, but sufficiently small to be safe to discuss in semiclassical gravity framework. Returning to the geometric units, the heat capacity of black holes can be obtained from

$$
C_{\mathrm{R}}=T_{\mathrm{R}}\left(\frac{\partial S_{\mathrm{R}}}{\partial T_{\mathrm{R}}}\right)=-\frac{2 \pi r_{h}^{2}}{1-\lambda \pi r_{h}^{2}}=-\frac{2 \pi r_{h}^{2}}{1-\frac{r_{h}^{2}}{L_{\lambda}^{2}}} .
$$

Notice that the heat capacity diverges at $r_{h}=L_{\lambda}$. This indicates the distinction between two phases of black holes in asymptotically flat spacetime, namely the unstable small black hole $\left(r_{h}<L_{\lambda}\right)$ with negative heat capacity $\left(C_{\mathrm{R}}<0\right)$ 
and the stable large black hole $\left(r_{h}>L_{\lambda}\right)$ with positive heat capacity $\left(C_{\mathrm{R}}>0\right)$. Therefore, the black holes can be in thermal equilibrium with infinite heat bath at a fixed temperature when the event horizon radius is larger than this characteristic length scale. This implies that the canonical ensemble exists for the Sch-flat from Rényi statistics.

The Hawking temperature of a black hole is minimum at $r_{h}=L_{\lambda}$, where it takes the form

$$
T_{\min }=\frac{1}{2} \sqrt{\frac{\lambda}{\pi}}=\frac{1}{2 \pi L_{\lambda}} .
$$

Below $T_{\min }$, a black hole is not allowed to be created due to the absence of a real value of $r_{h}$ as a solution of (18). Accordingly, this behavior of black holes in flat spacetime via Rényi statistics is in the same way as that of AdS black holes in the GB statistics approach [49]. Interestingly, the presence of $L_{\lambda}$ in the former case seems to be mathematically equivalent to that of the $\operatorname{AdS}$ radius $L_{\mathrm{AdS}}$ in the latter case. Moreover, the existence of this characteristic length scale, in both cases, is a sign of the presence of small/large black hole phase transition.

\section{GENERALIZED SMARR FORMULA}

In the standard GB statistics, the first law of black hole thermodynamics can be written in the form

$$
d M=T_{\mathrm{H}} d S_{\mathrm{BH}}+\Omega d J+\Phi d Q,
$$

where $\Omega$ is the horizon angular velocity, $J$ is the angular momentum, $\Phi$ is the horizon electric potential, and $Q$ is the black hole's electric charge. Applying Euler's theorem for homogeneous function to the first law of black hole thermodynamics, the black hole mass can be written in the form [50]

$$
M=2 T_{\mathrm{H}} S_{\mathrm{BH}}+2 \Omega J+\Phi Q .
$$

Note that this is the conventional Smarr formula within the framework of GB statistics. On the other hand, we can obtain a modified version of the Smarr formula from the Rényi statistics and treat the nonextensivity parameter $\lambda$ as a thermodynamic variable, which gives us the first law of black hole thermodynamics with the presence of the work term $P d V$. We will review shortly the process, as demonstrated in [22], to obtain this generalized Smarr formula in the following.

Considering a Schwarzschild black hole, we assume that the nonextensive parameter is sufficiently small to avoid reaching the quantum gravity limit, as discussed in the previous section. Thus, we may use $0<\lambda \ll 1$. Substituting the Bekenstein-Hawking entropy $S_{\mathrm{BH}}$ and Hawking temperature $T_{\mathrm{H}}$ into (9) and (10), we can obtain

$$
S_{\mathrm{BH}}=\frac{e^{\lambda S_{\mathrm{R}}}-1}{\lambda} \quad \text { and } \quad T_{\mathrm{H}}=\frac{T_{\mathrm{R}}}{e^{\lambda S_{\mathrm{R}}}} .
$$

We can reach a generalized Smarr formula within the Rényi statistics by putting (25) into the Smarr formula [50] and expanding it in the power series of the parameter $\lambda$. Then, we arrive at the formula

$$
M=2 T_{\mathrm{R}} S_{\mathrm{R}}-\lambda \frac{\pi r_{h}^{3}}{4}+\mathcal{O}\left(\lambda^{2}\right) .
$$

Here, we can introduce the thermodynamics pressure $P$ and the thermodynamic volume $V$ by identifying

$$
P=\frac{3 \lambda}{32}=\frac{3}{32 \pi L_{\lambda}^{2}}, \quad V=\frac{4}{3} \pi r_{h}^{3} .
$$

Keeping up to only the lowest order of $\lambda$, (26) comes to be in a form of the Smarr-like formula

$$
M=2 T_{\mathrm{R}} S_{\mathrm{R}}-2 P V .
$$

Note that the dimension of $M, P$, and $V$ in (27) and (28) coincides with the fact that $M \sim L, P \sim L^{-2}$, and $V \sim L^{3}$. We can call this relation in (28) a generalized Smarr formula via Rényi statistics.

As shown above, $\lambda$ has been treated as a dynamical variable; namely it is associated with the thermodynamic pressure $P$. This notion and its corresponding first law of black hole thermodynamics has been proposed in [22]. Following this approach, we substitute (25) into (23), then the first law of black hole thermodynamics in the Rényi extended phase space of the Schwarzschild black hole with the variation of $\lambda$ takes the form

$$
\begin{aligned}
d M & =\frac{T_{\mathrm{R}}}{e^{\lambda S_{\mathrm{R}}}} d\left(\frac{e^{\lambda S_{\mathrm{R}}}-1}{\lambda}\right), \\
& =T_{\mathrm{R}} d S_{\mathrm{R}}+\frac{1}{8} \pi r_{h}^{3} d \lambda+\mathcal{O}\left(\lambda^{2}\right) .
\end{aligned}
$$

Hence, keeping up to the first order in $\lambda$ and using the definition of $P$ and $V$ in (27), we arrive at the relation

$$
d M=T_{\mathrm{R}} d S_{\mathrm{R}}+V d P .
$$

This result shows that the mass $M=M\left(S_{\mathrm{R}}, P\right)$ is a function of $S_{\mathrm{R}}$ and $P$. This indicates that the black hole mass $M$ is interpreted as the enthalpy $H\left(S_{\mathrm{R}}, P\right)$ in the Rényi thermodynamics. As the quantity conjugate to the pressure, the thermodynamic volume can be obtained from the relation $V=\left(\frac{\partial M}{\partial P}\right)_{S_{\mathrm{R}}}$ at the first order of $\lambda$. Through the Legendre transformation $U=H-P V$, the first law of black hole thermodynamics extends to be in the form

$$
d U=T_{\mathrm{R}} d S_{\mathrm{R}}-P d V
$$


In this framework, the extended first law of black hole thermodynamics due to the presence of the emergence $P d V$ term in (31) becomes consistent with the first law of thermodynamics. Since the nonextensive nature of black holes results from long-range gravitational interactions, the degrees of freedom of black holes do not only interact among themselves in the system, but also interact with their environment. We have suggested in [22] that the correlations between black hole degrees of freedom and heat bath may appear as the additional work term $P d V$ in (31). On the other hand, the emergence of this work term can occur as a consequence of the cosmological constant in the AdS black hole with the GB statistics in the framework of extended phase space [29-33]. Intriguingly, this is the equivalence between the nonextensivity parameter $\lambda$ in Rényi-flat black holes and the cosmological constant $\Lambda$ in AdS via GB-AdS black holes.

\section{SOLID-LIQUID PHASE TRANSITION AND LATENT HEAT VIA THE RÉNYI EXTENDED PHASE SPACE}

To describe the thermal properties of gas, we use an equation of state to relate the thermodynamic quantities, such as pressure, volume, and temperature in a single equation. The extended first law of black hole thermodynamics (31) is very similar to a system of gas enclosed in the volume $V$ and subject to an external pressure $P$. From this similarity, we will construct an equation of state to explore thermodynamic properties and phase structure of a black hole by substituting the nonextensivity parameter $\lambda=\frac{32 P}{3}$ and the event horizon radius $r_{\mathrm{h}}=\left(\frac{3 V}{4 \pi}\right)^{\frac{1}{3}}$ into the temperature expression (18). Solving $P=P\left(T_{\mathrm{R}}, V\right)$, we obtain an equation of state of the Sch-flat black hole as

$$
P=\frac{1}{8}\left(\frac{4 \pi}{3}\right)^{\frac{1}{3}}\left(\frac{3 T_{\mathrm{R}}}{V^{\frac{1}{3}}}-\left(\frac{3}{4 \pi}\right)^{\frac{2}{3}} \frac{1}{V^{\frac{2}{3}}}\right)
$$

For this equation of state, the curves of fixed temperature are plotted in Fig. 3, where the pressure has a maximum value $P_{\max }$ at volume $V_{o}$ as

$$
P_{\max }=\frac{3 \pi}{8} T_{\mathrm{R}}^{2}, \quad V_{o}=\frac{1}{\left(6 \pi^{2}\right)^{\frac{1}{3}} T_{\mathrm{R}}} .
$$

Below $P_{\max }$, there are two black hole phases associated with small and large black hole configurations when $V<V_{o}$ and $V>V_{o}$, respectively. The Rényi entropy given in terms of $P$ and $V$ is

$$
S_{\mathrm{R}}(P, V)=\frac{3}{32 P} \ln \left(1+16\left(\frac{\pi}{6}\right)^{\frac{1}{3}} P V^{\frac{2}{3}}\right) .
$$

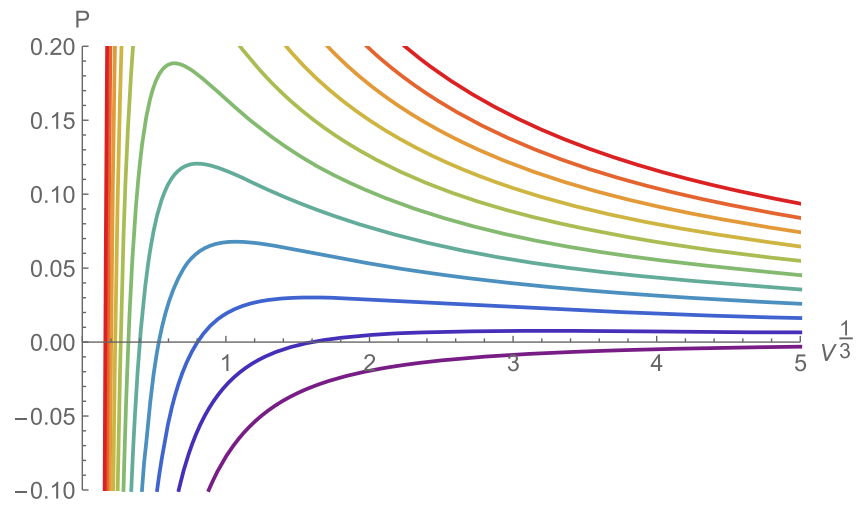

FIG. 3. The isothermal curves in the $P-V^{1 / 3}$ diagram of the Schflat in Rényi extended phase space is shown. The temperature decreases from top to bottom. At a given temperature, there are two phases of black holes, namely the small black hole and large black hole, which correspond to positive and negative slope in $P-V^{1 / 3}$ plane, respectively. The shape of isothermal curve in the $P-V$ plane is the same but more horizontally stretched.

The heat capacity at constant pressure can be expressed in the form

$$
C_{\mathrm{P}}=T_{\mathrm{R}}\left(\frac{\partial S_{\mathrm{R}}}{\partial T_{\mathrm{R}}}\right)_{P}=-3\left(\frac{\pi}{6}\right)^{\frac{1}{3}} \frac{V^{\frac{2}{3}}}{1-\left(\frac{V}{V_{c}}\right)^{\frac{2}{3}}},
$$

where $V_{c}=\frac{1}{32} \sqrt{\frac{3}{2 \pi}} \frac{1}{p^{\frac{3}{2}}}$. Obviously, the heat capacity is negative when $V<V_{c}$, positive when $V>V_{c}$, and diverges at $V=V_{c}$. In the large volume limit $V \rightarrow \infty$, the heat capacity converges to the positive finite value $C_{\mathrm{P}}=\frac{3}{16 P}$. The temperature is minimum at the volume $V_{c}$, which can be described in terms of the pressure $P$ as

$$
T_{\min }=\sqrt{\frac{8 P}{3 \pi}} .
$$

For $T<T_{\min }$, there is no black hole solution; the system is in the thermal radiation phase. This is illustrated in Fig. 4.

Thermal fluctuations can induce the microscopic degrees of freedom to rearrange, resulting in a phase transition of macroscopic state of matter. This interesting phenomena also occurs in gravitational physics, such as the nucleation of an AdS black hole in a thermal heat bath. The thermodynamic stability of the thermal phase under constant pressure and temperature can be described by Gibbs free energy. In a thermal gravitational system, the Gibbs free energy can be calculated from the standard Euclidean action method. Since we used Rényi statistics to describe the thermodynamics of a black hole, the on shell Gibbs free energy should be replaced by

$$
G_{\mathrm{R}}=M-T_{\mathrm{R}} S_{\mathrm{R}}
$$



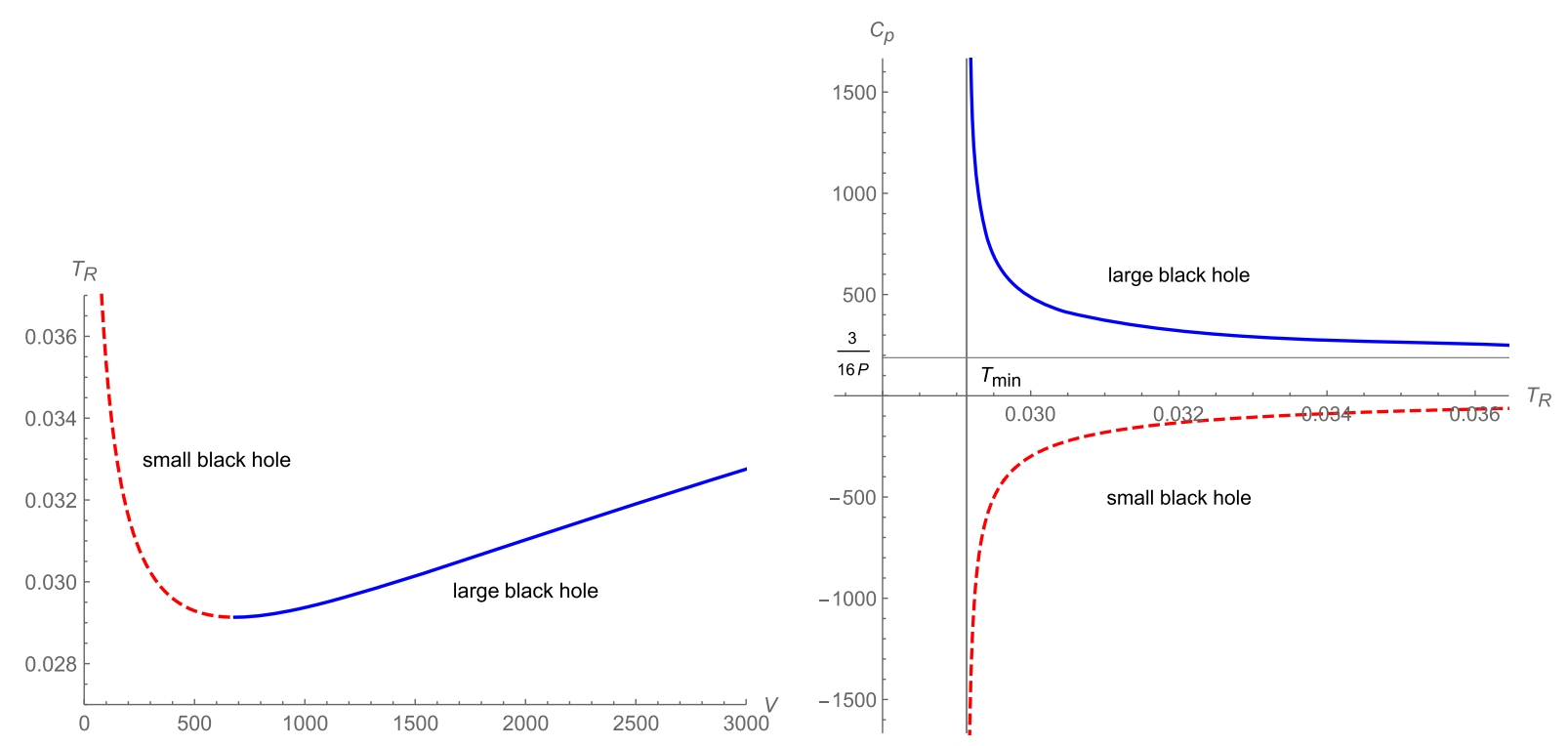

FIG. 4. Left: the Rényi temperature of a Schwarzschild black hole $T_{\mathrm{R}}$ versus the thermodynamic volume $V$ is plotted with $P=0.001$. Right: the corresponding heat capacity at constant pressure $C_{\mathrm{P}}$ of a black hole versus $T_{\mathrm{R}}$. In both panels, the dashed red and solid blue lines represent small and large black holes, respectively. The black hole solution does not exist when $T<T_{\min }$.

where $M$ is the enthalpy of the system. As mentioned in Sec. III, the canonical ensemble with fixed temperature exists in the consideration with Rényi statistics. Following from $[51,52]$, we can construct the off shell Gibbs free energy of the black hole in the canonical ensemble at fix temperature $T$ by substituting the black hole's temperature $T_{\mathrm{R}}$ in (37) with the ensemble temperature $T$. In this way, we obtain

$$
G_{\mathrm{R}}=\frac{1}{2}\left(\frac{3}{4 \pi}\right)^{\frac{1}{3}} V^{\frac{1}{3}}-\frac{3}{32} \frac{T}{P} \ln \left[1+16\left(\frac{\pi}{6}\right)^{\frac{1}{3}} P V^{\frac{2}{3}}\right] .
$$

Here, we have identified the thermodynamic volume $V$ as an order parameter. To obtain the black hole configuration in the heat bath, we replace the black hole's temperature $T_{\mathrm{R}}$ in (32) by the ensemble temperature $T$, then the order parameter $V$ of the small and large black hole can be solved analytically by

$$
V_{\mathrm{LBH} / \mathrm{SBH}}^{\frac{1}{3}}=\left(\frac{4 \pi}{3}\right)^{\frac{1}{3}} \frac{T}{2 \pi T_{\min }^{2}}\left(1 \pm \sqrt{1-\frac{T_{\min }^{2}}{T^{2}}}\right)
$$

where $V_{\mathrm{LBH}}$ and $V_{\mathrm{SBH}}$ represent the volume of large and small black holes, respectively. In the configuration where there is no black hole, namely, in the case where $r_{h}=0$, a Minkowski spacetime filled with thermal radiation represents classical hot flat space phase, which has the order parameter $V_{\text {rad }}=0$. Moreover, the Gibbs free energy and the entropy of this phase are virtually zero because the number of quantum particles associated with the radiation occupying the spacetime is very small.
The plots of the off shell and on shell Gibbs free energy are shown in Fig. 5, the Hawking-Page-like phase transition emerge in a similar way as the black hole in AdS space via GB statistics [49]. In a very low temperature limit $T<T_{\min }$, there no black hole exists since the order parameter of the black hole in (39) is an imaginary number. The only one global minimum of the $G_{R}$ curve is at $V_{\text {rad }}=0$, representing a pure thermal radiation in an asymptotically flat. Interestingly, ours result is in contrast to the GB statistics, which show that the black hole has no minimum bound on its temperature. If we increase the temperature until $T=T_{\min }$, the black hole nucleation starts here at an inflection point $V_{c}$ of $G_{\mathrm{R}}$.

For $T_{\min }<T<T_{\mathrm{HP}}$, the small and large black hole phases that emerged correspond to a local maximum and a local minimum of the $G_{\mathrm{R}}$ curve, respectively. However, the two local extremum has higher free energy than the thermal radiation phase, therefore the most thermodynamical preferred phase is still the pure thermal radiation state.

At $T=T_{\mathrm{HP}}$, the two local minimum are degenerate, namely the thermal radiation phase and the large black hole phase, suggesting a coexistence phase between them. The Hawking-Page temperature is determined from $G_{\mathrm{R}}=0$, which can be described numerically by

$$
T_{\mathrm{HP}} \sim 1.14 \sqrt{P} .
$$

The coexistence line of thermal radiation/large black hole phases can be written in the form

$$
\left.P\right|_{\text {coexistence }} \sim 0.769 T_{\mathrm{R}}^{2}
$$



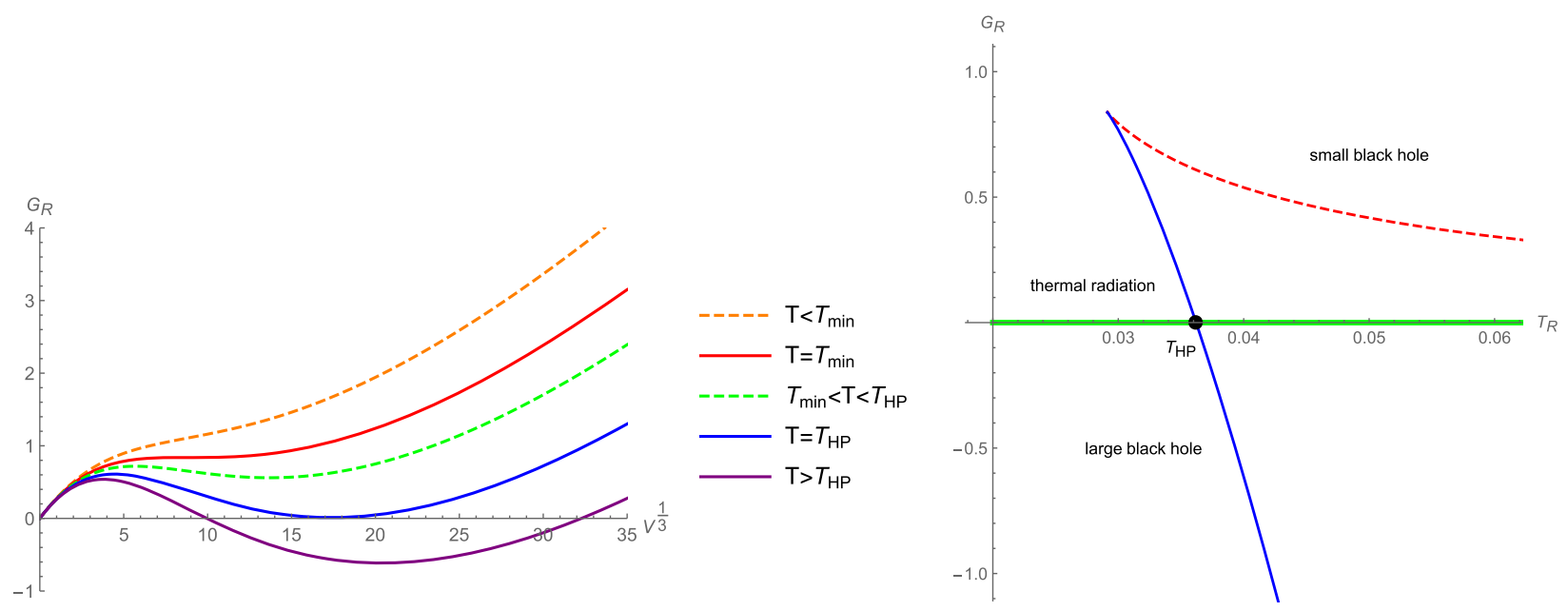

FIG. 5. Left: the off shell Gibbs free energy of a Schwarzschild black hole $G_{\mathrm{R}}$ versus the thermodynamic volume $V^{\frac{1}{3}}$ is plotted with $P=0.001$. Note that the curve of $G_{\mathrm{R}}$ versus $V$ looks similar to this graph, but its horizontal coordinate is much more extended. Right: the on shell Gibbs free energy $G_{\mathrm{R}}$ as a function of Rényi temperature $T_{\mathrm{R}}$ is shown.

The coexistence line in the $P-T_{\mathrm{R}}$ plane is plotted in Fig. 6 and has no termination point because $P$ in (40) has no bound, so the Hawking-Page phase transition can occur at all pressures. This is reminiscent of a solid-liquid phase transition [31,32]. When the system crosses this coexistence line, the order parameter $V_{\text {rad }}=0$ of thermal radiation phase jumps discontinuously to the large black hole phase

$$
V_{\mathrm{HP}} \sim \frac{0.168}{P^{\frac{3}{2}}}
$$

in the $G_{\mathrm{R}}$ curve, which is characteristic of the first-order phase transition. In this way, the entropy of the system (34) also change discontinuously, where it takes the form

$$
\Delta S=S_{\mathrm{LBH}}-S_{\mathrm{rad}}=\frac{3}{32 P} \ln \left(1+16\left(\frac{\pi}{6}\right)^{\frac{1}{3}} P V_{\mathrm{HP}}^{\frac{2}{3}}\right),
$$

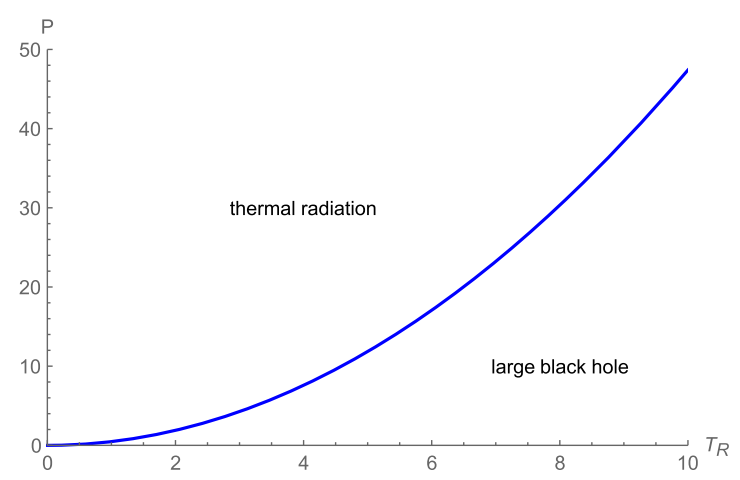

FIG. 6. The $P-T_{\mathrm{R}}$ phase diagram of the first order, the so-called Hawking-Page phase transition, between thermal radiation and large black hole via Rényi statistics. Note that the coexistence line continues extending and does not stop running at high pressure and temperature. This implies the absence of a critical point and then supports that this phase transition is similar to a solid-liquid phase transition, instead of a liquid-gas phase transition. where $S_{\text {rad }} \sim 0$. At the coexistence line, their Gibbs free energies are equal, i.e., $G_{\mathrm{rad}}\left(T_{\mathrm{HP}}\right)=G_{\mathrm{LBH}}\left(T_{\mathrm{HP}}\right)$, thus we obtain

$$
E_{\text {rad }}-T_{\mathrm{HP}} S_{\text {rad }}=M_{\mathrm{LBH}}-T_{\mathrm{HP}} S_{\mathrm{LBH}},
$$

where $E_{\text {rad }}$ is the energy of thermal radiations. From this, we then obtain

$$
\begin{aligned}
S_{\mathrm{LBH}}-S_{\mathrm{rad}} & =\frac{M_{\mathrm{LBH}}-E_{\mathrm{rad}}}{T_{\mathrm{HP}}}, \\
\Delta S & =\frac{L}{T_{\mathrm{HP}}},
\end{aligned}
$$

where we have replaced $M_{\mathrm{LBH}}-E_{\mathrm{rad}}$ by the latent heat $L$ of the transition to nucleate the large black hole in flat spacetime from the pure thermal radiations and vice versa. This can be done because the latent heat is usually defined by

$$
L=T_{\mathrm{HP}} \Delta S \text {. }
$$

By substituting (40), (42), and (43) into (45), we obtain

$$
L \sim \frac{0.171}{\sqrt{P}} .
$$

Notice that it is equal to the black hole's mass on the coexistence line $M_{\mathrm{HP}}=\frac{1}{2}\left(\frac{3 V_{\mathrm{HP}}}{4 \pi}\right)^{\frac{1}{3}}$. Our result is very similar to the black hole in AdS from GB statistics $[53,54]$. In a case of the Sch-flat with GB statistics, the pressure $P=0$, the latent heat tends to be infinite, which means that the thermal radiation cannot classically nucleate to form a black hole in asymptotically flat spacetime. Finally, at the high temperature limit $T>T_{\mathrm{HP}}$, the large black hole phase 
is the most thermodynamically preferred state since its $G_{\mathrm{R}}$ becomes the absolute globally minimum value.

\section{BLACK HOLE HEAT ENGINE}

We have already discussed the generalized Smarr formula via Rényi statistics in Sec. IV, which allows the work term to emerge in the first law of black hole thermodynamics. In this section, we will investigate further the second law of black hole thermodynamics within the Rényi extended phase space approach. To achieve this, we study the Carnot heat engine by using the black hole as a working substance. As stated in the Carnot theorem, every reversible heat engine performing between hot and cold energy reservoirs has the same efficiency, regardless of the type of engine and working substance. One question may be whether, within the Rényi extended thermodynamics, the black hole heat engine can give the Carnot efficiency.

Let us start with reviewing some theoretical backgrounds about heat engines. Generically, a cyclic thermodynamic process operates between the hot and the cold heat reservoir with the temperature $T_{\mathrm{H}}$ and $T_{\mathrm{C}}$, respectively. In each cycle, it performs the net work $W_{\text {eng }}$, whereas it absorbs the heat from the hot reservoir $\left|Q_{\mathrm{H}}\right|$ and expels the heat to the cold reservoir $\left|Q_{\mathrm{C}}\right|$. From the first law of thermodynamics, the thermal efficiency of a heat engine is in the form

$$
\eta=\frac{W_{\text {eng }}}{\left|Q_{\mathrm{H}}\right|}=1-\frac{\left|Q_{\mathrm{C}}\right|}{\left|Q_{\mathrm{H}}\right|},
$$

which is always less than 1 , according to the second law of thermodynamics. However, the theoretical maximum efficiency, namely the Carnot efficiency, can be obtained by applying the second law of thermodynamics. We will demonstrate it here.

Consider the total change of entropy, including the system and environment. Through each cycle, the total entropy is $\Delta S_{\text {total }}=\Delta S_{\text {eng }}+\Delta S_{\text {env }}$. The change of environment consists of the hot and cold heat reservoirs. Therefore, the change of environment entropy is the sum of the change of the entropy of hot and cold heat reservoirs, that is, $\Delta S_{\mathrm{H}}$ and $\Delta S_{\mathrm{C}}$, respectively. Since the cyclic process here is the reversible process, we have $\Delta S_{\text {eng }}=0$ after each cycle finishes. The total entropy $\Delta S_{\text {total }}$ is reduced to be only in the term of $\Delta S_{\text {env }}$. Hence, the second law reads

$$
\Delta S_{\text {total }}=\Delta S_{\mathrm{H}}+\Delta S_{\mathrm{C}} \geq 0
$$

From the formula $\Delta S=\frac{\Delta Q}{T}$, the entropy change of the hot and cold heat reservoirs is $\Delta S_{\mathrm{H}}=-\frac{\left|Q_{\mathrm{H}}\right|}{T_{\mathrm{H}}}$ and $\Delta S_{\mathrm{C}}=\frac{\left|Q_{\mathrm{C}}\right|}{T_{\mathrm{C}}}$, respectively. Substituting them into (48), we have

$$
\begin{aligned}
-\frac{\left|Q_{\mathrm{H}}\right|}{T_{\mathrm{H}}}+\frac{\left|Q_{\mathrm{C}}\right|}{T_{\mathrm{C}}} & \geq 0, \\
-\frac{\left|Q_{\mathrm{H}}\right|}{T_{\mathrm{H}}}+\frac{\left|Q_{\mathrm{H}}\right|-W_{\mathrm{BH}}}{T_{\mathrm{C}}} & \geq 0, \\
\frac{W_{\mathrm{BH}}}{\left|Q_{\mathrm{H}}\right|} & \leq 1-\frac{T_{\mathrm{C}}}{T_{\mathrm{H}}}, \\
\eta & \leq 1-\frac{T_{\mathrm{C}}}{T_{\mathrm{H}}} .
\end{aligned}
$$

Therefore, the maximum value of $\eta$ is $\eta_{\mathrm{C}}=1-\frac{T_{\mathrm{C}}}{T_{\mathrm{H}}}$. The $\eta_{\mathrm{C}}$ is the efficiency of the heat engine as it operates along the Carnot cycle. Carnot's theorem can be stated as "no heat engine operating between two heat reservoirs can be more efficient than a reversible heat engine operating between the same two reservoirs." Obviously, the two statements, Carnot's theorem and the GSL, are equivalent.

Here, the black hole can be in thermal equilibrium with an infinite heat reservoir. Basically, this is a condition necessary for the existence of a quasistatic change in the reversible process and the emergence of mechanical work from heat via the $P d V$ term in (31). We can construct the heat engine operating through the Carnot cycle in the context of an asymptotically flat black hole. Before mentioning the Carnot cycle of a black hole, it is important to derive some useful relations of the internal energy from the equation of state (32). Let us consider the first law of black hole thermodynamics (31)

$$
T_{\mathrm{R}} d S_{\mathrm{R}}=d U+P d V
$$

The Rényi entropy can be written in the form $S_{\mathrm{R}}=$ $S_{\mathrm{R}}\left(T_{\mathrm{R}}, V\right)$, therefore its differential form is given by

$$
d S_{\mathrm{R}}=\left(\frac{\partial S_{\mathrm{R}}}{\partial T_{\mathrm{R}}}\right)_{V} d T_{\mathrm{R}}+\left(\frac{\partial S_{\mathrm{R}}}{\partial V}\right)_{T_{\mathrm{R}}} d V
$$

Multiplying both sides of the above equation by $T_{\mathrm{R}}$ and using the Maxwell relation, $\left(\frac{\partial S_{\mathrm{R}}}{\partial V}\right)_{T_{\mathrm{R}}}=\left(\frac{\partial P}{\partial T_{\mathrm{R}}}\right)_{V}$, we obtain

$$
T_{\mathrm{R}} d S_{\mathrm{R}}=C_{\mathrm{V}} d T_{\mathrm{R}}+T_{\mathrm{R}}\left(\frac{\partial P}{\partial T_{\mathrm{R}}}\right)_{V} d V
$$

where $C_{\mathrm{V}}=T_{\mathrm{R}}\left(\frac{\partial S_{\mathrm{R}}}{\partial T_{\mathrm{R}}}\right)_{V}$, that is, the heat capacity at fixed constant volume. Combining (50) and (52), the variation of internal energy becomes

$$
d U=C_{\mathrm{V}} d T_{\mathrm{R}}+\left[T_{\mathrm{R}}\left(\frac{\partial P}{\partial T_{\mathrm{R}}}\right)_{V}-P\right] d V .
$$

Because $d U$ is an exact differential equation, we have 


$$
\frac{\partial C_{\mathrm{V}}}{\partial V}=\frac{\partial}{\partial T_{\mathrm{R}}}\left[T_{\mathrm{R}}\left(\frac{\partial P}{\partial T_{\mathrm{R}}}\right)_{V}-P\right]=T_{\mathrm{R}}\left(\frac{\partial^{2} P}{\partial T_{\mathrm{R}}^{2}}\right)_{V} .
$$

From the equation of state in (32), we found that $\left(\frac{\partial^{2} P}{\partial T_{\mathrm{R}}^{2}}\right)_{V}=0$; this means that $C_{\mathrm{V}}$ does not depend on the thermodynamics volume $V$ and depends only on the temperature $T_{\mathrm{R}}$ [55-57]. Substituting $d U$ from (53) into (50),

$$
\begin{aligned}
d Q & =d U+P d V \\
& =C_{\mathrm{V}} d T_{\mathrm{R}}+T_{\mathrm{R}}\left(\frac{\partial P}{\partial T_{\mathrm{R}}}\right)_{V} d V \\
& =C_{\mathrm{V}} d T_{\mathrm{R}}+\frac{3}{8}\left(\frac{4 \pi}{3}\right)^{\frac{1}{3}} \frac{T_{\mathrm{R}}}{V^{\frac{1}{3}}} d V,
\end{aligned}
$$

where $d Q=T_{\mathrm{R}} d S_{\mathrm{R}}$. Note that we used an equation of state (32) to obtain the second term of (55).

We consider the black hole as a working substance that operates between hot and cold reservoirs with the temperatures $T_{\mathrm{H}}$ and $T_{\mathrm{C}}$, respectively. One of the basic Carnot cycles consists of the four steps as shown in Fig. 3. In the isothermal process $A \rightarrow B$, the heat engine performs the mechanical work the isothermal expansion, whereas it absorbs the heat from the energy reservoir at the temperature $T_{\mathrm{H}}$. The relation between the absorbed heat $Q_{\mathrm{H}}$ and $T_{\mathrm{H}}$ and the volume $V_{\mathrm{A}}$ and $V_{\mathrm{B}}$ can be obtained by integrating (55) as follows:

$$
\begin{aligned}
Q_{\mathrm{H}} & =\int_{A}^{B} d Q \\
& =\frac{3}{8}\left(\frac{4 \pi}{3}\right)^{\frac{1}{3}} T_{\mathrm{H}} \int_{V_{\mathrm{A}}}^{V_{\mathrm{B}}} \frac{d V}{V^{\frac{1}{3}}} \\
& =\frac{9}{16}\left(\frac{4 \pi}{3}\right)^{\frac{1}{3}} T_{\mathrm{H}}\left(V_{\mathrm{B}}^{\frac{2}{3}}-V_{\mathrm{A}}^{\frac{2}{3}}\right),
\end{aligned}
$$

where the first term of (55) is zero since $d T_{\mathrm{R}}=0$ along the isothermal path. In a similar way, the isothermal compression process $C \rightarrow D$ in contact with the cold energy reservoir of the temperature $T_{\mathrm{C}}$ exhausts the heat of the form

$$
\begin{aligned}
Q_{\mathrm{C}} & =\int_{C}^{D} d Q \\
& =-\frac{9}{16}\left(\frac{4 \pi}{3}\right)^{\frac{1}{3}} T_{\mathrm{C}}\left(V_{\mathrm{C}}^{\frac{2}{3}}-V_{\mathrm{D}}^{\frac{2}{3}}\right) .
\end{aligned}
$$

Manifestly, since $V_{\mathrm{B}}>V_{\mathrm{A}}$ and $V_{\mathrm{C}}>V_{\mathrm{D}}$, it can be seen from (56) and (57) that the heat $Q_{\mathrm{H}}>0$ and $Q_{\mathrm{C}}<0$.

On the other hand, the path $B \rightarrow C$ and $D \rightarrow A$ are adiabatic processes, along which $d Q=0$. Integrating (55) for these two adiabatic processes, we have

$$
\int_{T_{\mathrm{R}, \mathrm{B}}}^{T_{\mathrm{R}, \mathrm{C}}} \frac{C_{\mathrm{V}}}{T_{\mathrm{R}}} d T_{\mathrm{R}}+\frac{9}{16}\left(\frac{4 \pi}{3}\right)^{\frac{1}{3}}\left(V_{\mathrm{C}}^{\frac{2}{3}}-V_{\mathrm{B}}^{\frac{2}{3}}\right)=0,
$$

and

$$
\int_{T_{\mathrm{R}, \mathrm{D}}}^{T_{\mathrm{R}, \mathrm{A}}} \frac{C_{\mathrm{V}}}{T_{\mathrm{R}}} d T_{\mathrm{R}}+\frac{9}{16}\left(\frac{4 \pi}{3}\right)^{\frac{1}{3}}\left(V_{\mathrm{A}}^{\frac{2}{3}}-V_{\mathrm{D}}^{\frac{2}{3}}\right)=0 .
$$

Combining (58) and (59), we obtain the condition

$$
V_{\mathrm{C}}^{\frac{2}{3}}-V_{\mathrm{B}}^{\frac{2}{3}}=-\left(V_{\mathrm{A}}^{\frac{2}{3}}-V_{\mathrm{D}}^{\frac{2}{3}}\right) \quad \text { or } \quad V_{\mathrm{C}}^{\frac{2}{3}}-V_{\mathrm{D}}^{\frac{2}{3}}=V_{\mathrm{B}}^{\frac{2}{3}}-V_{\mathrm{A}}^{\frac{2}{3}} .
$$

Since $\frac{C_{\mathrm{V}}}{T_{\mathrm{R}}}$ depends only on the temperature and $T_{\mathrm{R}, \mathrm{A}}=$ $T_{\mathrm{R}, \mathrm{B}}=T_{\mathrm{H}}$ and $T_{\mathrm{R}, \mathrm{C}}=T_{\mathrm{R}, \mathrm{D}}=T_{\mathrm{C}}$, we arrive at the formula

$$
\int_{T_{\mathrm{R}, \mathrm{B}}}^{T_{\mathrm{R}, \mathrm{C}}} \frac{C_{\mathrm{V}}}{T_{\mathrm{R}}} d T_{\mathrm{R}}+\int_{T_{\mathrm{R}, \mathrm{D}}}^{T_{\mathrm{R}, \mathrm{A}}} \frac{C_{\mathrm{V}}}{T_{\mathrm{R}}} d T_{\mathrm{R}}=0 .
$$

The thermal efficiency of the Carnot engine using the Sch-flat black hole as a working substance $\eta_{c}$ can be obtained by substituting (56) and (57) into (47) and using the relation (60). Hence, we obtain the thermal efficiency

$$
\eta_{c}=1-\frac{\left|Q_{\mathrm{C}}\right|}{\left|Q_{\mathrm{H}}\right|}=1-\frac{T_{\mathrm{C}}}{T_{\mathrm{H}}} .
$$

Interestingly, the equation of state of the Sch-flat black hole within the Rényi extended phase space approach gives rise to the thermal efficiency in the reversible process $\eta_{c}$ following Carnot's theorem in the same way as occurs in the conventional matter. This implies that no black hole heat engine can convert heat from a hot reservoir into mechanical work without some energy released to the environment. Therefore, the Rényi extended phase space approach in black hole thermodynamics renders the thermodynamic description satisfying the second law of thermodynamics or the GSL. Remarkably, our results have given further evidence that treating the nonextensivity parameter $\lambda$ as the thermodynamic pressure $P$, as proposed in [22], surprisingly brings about a consistent thermodynamics.

\section{CONCLUSION AND DISCUSSION}

After reviewing the nonextensive entropy approach in black hole thermodynamics in Secs. II-IV, we explore the Rényi thermodynamics of the Schwarzschild black hole in asymptotically flat spacetime in Sec. V as the first part of our study. The results indicate that, with $0<\lambda<1$, there are small and large black hole phases emerging, while this cannot occur in the GB statistics. Having introduced $L_{\lambda}=1 / \sqrt{\pi \lambda}$ as the nonextensivity length scale in [22], we find here that, as long as the black hole has the size $r_{h}$ 
greater than this length scale, it has positive heat capacity. Namely, the large black hole can be in thermal equilibrium with thermal radiation at a fixed temperature. This implies that the canonical ensemble can exist in consideration with this alternative Rényi entropy.

Treating the nonextensivity parameter $\lambda$ as a thermodynamic variable, it can be assigned as a thermodynamic pressure, whereas its conjugate variable is a thermodynamic volume. In this extended approach, the mass of a black hole represents the enthalpy rather than the internal energy. Interestingly, the mechanical work $P d V$ term exists in the first law of black hole thermodynamics (31), which allows us to construct the black hole heat engine, as discussed in this paper. We write the equation of state in terms of pressure $P$ and volume $V$ and study the thermal phase structure of the Sch-flat black hole in this Rényi extended phase space approach. The black hole exhibits the first-order Hawking-Page phase transition between the thermal radiation phase and large black hole phase, which can be determined from off shell Gibbs free energy. The coexistence curve between thermal radiation and large black hole in the $P-T$ plane is equivalent to the semiinfinite quadratic curve, which is reminiscent of a solidliquid phase transition. The latent heat of fusion from solid (corresponding to thermal radiation) to liquid (corresponding to large black hole) is inversely proportional to the square root of pressure, i.e., $L \sim 1 / \sqrt{P}$. We can deduce from this that the phase of thermal radiations cannot classically turn into the situation of a black hole nucleation within the GB statistics where $P=0$.

In the second part of our study in this paper, i.e., Sec. VI, we investigate the validity of the GSL in the context of the Rényi extended phase space approach. Interestingly, the quasistatic change of the black hole thermodynamical system in the reversible process is possible in this approach because the black hole can be in thermal equilibrium with its surroundings and there exists the emerging mechanical work from heat via the $P d V$ term. Consequently, we can construct a heat engine by taking the Sch-flat black hole as a working substance. We define a Carnot cycle in the $P-V$ plane and derive the thermal efficiency of a Sch-flat black hole heat engine. Intriguingly, we obtain the efficiency in the form $\eta_{c}=1-T_{\mathrm{C}} / T_{\mathrm{H}}$. This maximum possible efficiency of a reversible heat engine follows the second law since it gives the result corresponding to the Carnot theorem in the usual GB thermodynamics. In other words, the second law of thermodynamics or GSL is still satisfied even in the alternative Rényi thermodynamics. This gives more evidence that interpreting the parameter $\lambda$ as thermodynamic pressure and treating its conjugate as volume could provide a proper thermodynamic description.

In this work, our motivation is to demonstrate that the Rényi extended phase space approach may be a reasonable statistical model of black holes by considering some macroscopic phenomena including the thermal phase transition and the reversible heat engine. These are some embodiments that our consideration is in a well-behaved thermodynamic description, especially satisfying the second law of thermodynamics. However, we did not so far discuss the microscopic description or quantum originated aspects of the Rényi statistics approach to black holes thermodynamics. Let us spend the space here to discuss some potentially physical implications of the Rényi model in describing the quantum aspects of black holes.

Generically, in the long-range interaction systems like a self-gravitating system, their microstates could be correlated in some way with the heat reservoir. As we suggested in [22], some energy density emerging from the nontrivial correlation between a black hole and heat reservoir may be encoded in the nonextensive parameter $\lambda$. This emergent energy density from the correlations induces a pressure to the black holes in the same way as the cosmological constant in the case of AdS black holes through the extended phase space approach. It is important to emphasize that the appearance of this energy density in black hole thermodynamics is an emergent phenomena, whereas there is no contribution explicitly in the geometry of a classical black hole solution. Therefore, there is no direct mathematical parallel between geometric and thermodynamic descriptions in black hole thermodynamics from the Rényi statistics, which is different from the results of the consideration using the GB statistics.

To support the idea that the modified thermodynamics from Rényi statistics results from the correlations between a black hole and its environment, let us consider the scaling of the black hole's Rényi entropy, as shown in (17), with the horizon radius in the limit of small and large black holes, respectively, as follows:

$$
S_{\mathrm{R}}= \begin{cases}\pi r_{h}^{2}, & \text { if } r_{h} \ll L_{\lambda}, \\ \frac{2}{\lambda} \ln \left(\frac{r_{h}}{L_{\lambda}}\right), & \text { if } r_{h} \gg L_{\lambda} .\end{cases}
$$

In the $r_{h} \ll L_{\lambda}$ (small black hole) limit, it is obvious that the Rényi entropy approximately obeys an area law as the Bekenstein-Hawking entropy in the GB statistics. The area scaling of a black hole's entropy in this $\lambda \rightarrow 0$ limit may imply that the entropy corresponds to an entanglement entropy [4-6]. What about the logarithmic scaling of the black hole entropy in the $r_{h} \gg L_{\lambda}$ (large black hole) limit? Apparently, there is a violation of the area law when the horizon radius is much larger than the nonextensivity length scale. This logarithmic scaling entropy is reminiscent of some lattice models in condensed matter physics, which includes, for example, a one-dimensional quantum spin chain just away from criticality with the entanglement entropy of the form [58,59]

$$
S_{\mathrm{A}}=\frac{c}{6} \mathcal{A} \ln \frac{\xi}{a} .
$$


Note that $\xi$ is a correlation length, $a$ is a lattice spacing, and $\mathcal{A}$ is the number of boundary points between the system $\mathrm{A}$ and its complement $\mathrm{B}$. The expression above can be obtained from the von Neumann entropy of the subsystem A, where the degrees of freedom of the inaccessible subsystem B is traced out. Comparing this system with the Sch-flat one, the black hole analog of B could be the interior region covered by the event horizon in which the information cannot be reachable. On the other hand, the subsystem A may correspond to an exterior region outside the event horizon where an observer is sitting. Matching (63) in the large black hole limit with (64), we may identify that $r_{h}$ and $L_{\lambda}$ are associated with the correlation length $\xi$ and the lattice spacing $a$, respectively. Thus, these could be written symbolically as

$$
\begin{aligned}
& r_{h} \leftrightarrow \xi, \\
& L_{\lambda} \leftrightarrow a,
\end{aligned}
$$

where $\lambda$ may be related to the central charge $c$ in the form of $\lambda=12 / c \mathcal{A}$. As shown in Wolf et al. [60], considering the mutual information between subsystems leads to the conclusion that the area law of entropy emerges in the system with a short-range correlation; i.e., $\xi$ is small, while the area law is violated as $\xi$ becomes infinite. In this way, the logarithmic scaling of a black hole's entropy from the Rényi statistics as $r_{h} / L_{\lambda} \rightarrow \infty$, corresponding to $\xi / a \rightarrow \infty$, may imply the existence of long-range correlations between the black hole and its surroundings in the large black hole limit. It is interesting to note that the nonextensivity length scale $L_{\lambda}$ works as a lattice spacing in spacetime, which should be larger than the Planck length $l_{P}$ as discussed in (19). Therefore, the role of $L_{\lambda}$ may be a kind of the fine- or coarse-graining parameter for the entropy. Remarkably, these tend to indicate that the role of correlations in black hole thermodynamics emerging in the Rényi thermodynamic description may be originated from microscopic quantum aspects, which is interesting and to be explored further. Some hints could be provided by the works of applying long-range quantum gravity states to describe black holes $[61,62]$.

Recently, there have been many attempts to test classical general relativity and black hole thermodynamics, e.g., the gravitational wave experiments $[63,64]$ and analog gravity systems [65-67]; also see [68] for a review. It may be worth in some ways to explore these phenomena using the Rényi statistics and investigate whether there is any deviation from the standard GB statistics. This could indicate the nature of correlations in the system.

\section{ACKNOWLEDGMENTS}

We are grateful to Supakchai Ponglertsakul, Krai Cheamsawat, and Tanapat Deesuwan for helpful discussions. This publication has been supported by the Petchra Pra Jom Klao Ph.D. Research Scholarship from King Mongkut's University of Technology Thonburi (KMUTT) and the basic research fund 2021 from Thailand Science Research and Innovation (TSRI).
[1] J. M. Bardeen, B. Carter, and S. W. Hawking, The four laws of black hole mechanics, Commun. Math. Phys. 31, 161 (1973).

[2] J. D. Bekenstein, Black holes and entropy, Phys. Rev. D 7, 2333 (1973).

[3] S. W. Hawking, Particle creation by black Holes, Commun. Math. Phys. 43, 199 (1975); 46, 206(E) (1976).

[4] L. Bombelli, R. K. Koul, J. Lee, and R. D. Sorkin, A quantum source of entropy for black holes, Phys. Rev. D 34, 373 (1986).

[5] M. Srednicki, Entropy and Area, Phys. Rev. Lett. 71, 666 (1993).

[6] J. Eisert, M. Cramer, and M. B. Plenio, Area laws for the entanglement entropy—a review, Rev. Mod. Phys. 82, 277 (2010).

[7] S. W. Hawking, Black holes and thermodynamics, Phys. Rev. D 13, 191 (1976).

[8] T. Padmanabhan, Statistical mechanics of gravitating systems, Phys. Rep. 188, 285 (1990).

[9] C. Tsallis, Possible generalization of Boltzmann-Gibbs statistics, J. Stat. Phys. 52, 479 (1988).
[10] A. Rényi, On the dimension and entropy of probability distributions, Acta Math. Acad. Sci. Hung. 10, 193 (1959).

[11] T. S. Biró, G. G. Barnaföldi, and P. Van, Quark-gluon plasma connected to finite heat bath, Eur. Phys. J. A 49, 110 (2013).

[12] C. Y. Wong, G. Wilk, L. J. L. Cirto, and C. Tsallis, From QCD-based hard-scattering to nonextensive statistical mechanical descriptions of transverse momentum spectra in high-energy $p p$ and $p \bar{p}$ collisions, Phys. Rev. D 91, 114027 (2015).

[13] A. Deppman, E. Megías, and D. P. Menezes, Fractal structure of Yang-Mills fields, Phys. Scr. 95, 094006 (2020).

[14] J. A. S. Lima, R. Silva, and A. R. Plastino, Nonextensive Thermostatistics and the H Theorem, Phys. Rev. Lett. 86, 2938 (2001).

[15] T. S. Biró and E. Molnár, Non-extensive statistics, relativistic kinetic theory and fluid dynamics, Eur. Phys. J. A 48, 172 (2012).

[16] S. Mitra, Thermodynamics and relativistic kinetic theory for q-generalized Bose-Einstein and Fermi-Dirac systems, Eur. Phys. J. C 78, 66 (2018). 
[17] S. Thurner, R. Hanel, and P. Klimek, Introduction to the Theory of Complex Systems (Oxford University Press, Oxford, UK, 2018).

[18] C. Tsallis, Introduction to Nonextensive Statistical Mechanics: Approaching a Complex World (Springer Science +Business Media, New York, NY, USA, 2009).

[19] C. Tsallis and L. J. L. Cirto, Black hole thermodynamical entropy, Eur. Phys. J. C 73, 2487 (2013).

[20] V. G. Czinner and H. Iguchi, Rényi entropy and the thermodynamic stability of black holes, Phys. Lett. B 752, 306 (2016).

[21] V. G. Czinner and H. Iguchi, Thermodynamics, stability and Hawking-Page transition of Kerr black holes from Rényi statistics, Eur. Phys. J. C 77, 892 (2017).

[22] C. Promsiri, E. Hirunsirisawat, and W. Liewrian, Thermodynamics and van der Waals phase transition of charged black holes in flat spacetime via Rényi statistics, Phys. Rev. D 102, 064014 (2020).

[23] L. Tannukij, P. Wongjun, E. Hirunsirisawat, T. Deesuwan, and C. Promsiri, Thermodynamics and phase transition of spherically symmetric black hole in de Sitter space from Rényi statistics, Eur. Phys. J. Plus 135, 500 (2020).

[24] R. Nakarachinda, L. Tannukij, P. Wongjun, and E. Hirunsirisawat, Effective thermodynamical system of Schwarzschild-de Sitter black hole from Rényi statistics, arXiv:2106.02838.

[25] A. Chamblin, R. Emparan, C. V. Johnson, and R. C. Myers, Charged AdS black holes and catastrophic holography, Phys. Rev. D 60, 064018 (1999).

[26] A. Chamblin, R. Emparan, C. V. Johnson, and R. C. Myers, Holography, thermodynamics and fluctuations of charged AdS black holes, Phys. Rev. D 60, 104026 (1999).

[27] C. Niu, Y. Tian, and X. N. Wu, Critical phenomena and thermodynamic geometry of RN-AdS black holes, Phys. Rev. D 85, 024017 (2012).

[28] P. Burikham and C. Promsiri, The mixed phase of charged AdS black holes, Adv. High Energy Phys. 2016, 5864672 (2016).

[29] D. Kastor, S. Ray, and J. Traschen, Enthalpy and the mechanics of AdS black holes, Classical Quantum Gravity 26, 195011 (2009).

[30] D. Kubiznak and R. B. Mann, P-V criticality of charged AdS black holes, J. High Energy Phys. 07 (2012) 033.

[31] D. Kubiznak and R. B. Mann, Black hole chemistry, Can. J. Phys. 93, 999 (2015).

[32] D. Kubiznak, R. B. Mann, and M. Teo, Black hole chemistry: Thermodynamics with lambda, Classical Quantum Gravity 34, 063001 (2017).

[33] N. Altamirano, D. Kubiznak, R. B. Mann, and Z. Sherkatghanad, Thermodynamics of rotating black holes and black rings: Phase transitions and thermodynamic volume, Galaxies 2, 89 (2014).

[34] J. D. Bekenstein, Generalized second law of thermodynamics in black hole physics, Phys. Rev. D 9, 3292 (1974).

[35] S. W. Hawking, Gravitational Radiation from Colliding Black Holes, Phys. Rev. Lett. 26, 1344 (1971).

[36] R. M. Wald, The thermodynamics of black holes, Living Rev. Relativity 4, 6 (2001).

[37] A.C. Wall, Ten proofs of the generalized second law, J. High Energy Phys. 06 (2009) 021.
[38] C. V. Johnson, Holographic heat engines, Classical Quantum Gravity 31, 205002 (2014).

[39] A. Chakraborty and C. V. Johnson, Benchmarking black hole heat engines, I, Int. J. Mod. Phys. D 27, 1950012 (2018).

[40] A. Chakraborty and C. V. Johnson, Benchmarking black hole heat engines, II, Int. J. Mod. Phys. D 27, 1950006 (2018).

[41] C. V. Johnson, Gauss-Bonnet black holes and holographic heat engines beyond large $N$, Classical Quantum Gravity 33, 215009 (2016).

[42] C. V. Johnson, Born-Infeld AdS black holes as heat engines, Classical Quantum Gravity 33, 135001 (2016).

[43] B. Chandrasekhar and P. K. Yerra, Heat engines for dilatonic Born-Infeld black holes, Eur. Phys. J. C 77, 534 (2017).

[44] J. X. Mo and G. Q. Li, Holographic heat engine within the framework of massive gravity, J. High Energy Phys. 05 (2018) 122.

[45] J. Zhang, Y. Li, and H. Yu, Thermodynamics of charged accelerating AdS black holes and holographic heat engines, J. High Energy Phys. 02 (2019) 144.

[46] L.E. Reichl, A Modern Course in Statistical Physics, 4th revised and updated edition (Wiley-VCH Verlag GmbH and Co. KGaA, Weinheim, Germany, 2016).

[47] S. Abe, General pseudoadditivity of composable entropy prescribed by the existence of equilibrium, Phys. Rev. E 63, 061105 (2001).

[48] T. S. Biró and P. Ván, Zeroth law compatibility of nonadditive thermodynamics, Phys. Rev. E 83, 061147 (2011).

[49] S. W. Hawking and D. N. Page, Thermodynamics of black holes in anti-de Sitter space, Commun. Math. Phys. 87, 577 (1983).

[50] L. Smarr, Mass Formula for Kerr Black Holes, Phys. Rev. Lett. 30, 71 (1973); 30, 521(E) (1973).

[51] E. Spallucci and A. Smailagic, Maxwell's equal area law and the Hawking-Page phase transition, J. Gravity 2013, 525696 (2013).

[52] R. Li and J. Wang, Thermodynamics and kinetics of Hawkingpage phase transition, Phys. Rev. D 102, 024085 (2020).

[53] B. P. Dolan, Vacuum energy and the latent heat of AdS-Kerr black holes, Phys. Rev. D 90, 084002 (2014).

[54] A. Belhaj, M. Chabab, H. El Moumni, K. Masmar, M. B. Sedra, and A. Segui, On heat properties of AdS black holes in higher dimensions, J. High Energy Phys. 05 (2015) 149.

[55] D. C. Agraval and V. J. Menon, The Carnot cycle with the van der Waals equation of state, Eur. J. Phys 11, 88 (1990).

[56] M. N. Berberan-Santos, E. N. Bodunov, and L. Pogliani, The van der Waals equation: Analytical and approximate solution, J. Math. Chem. 43, 1437 (2008).

[57] P. C. Tjiang and S. H. Sutanto, The efficiency of the Carnot cycle with arbitrary gas equations of state, Eur. J. Phys. 27, 719 (2006).

[58] G. Vidal, J. I. Latorre, E. Rico, and A. Kitaev, Entanglement in Quantum Critical Phenomena, Phys. Rev. Lett. 90, 227902 (2003).

[59] P. Calabrese and J. L. Cardy, Entanglement entropy and quantum field theory, J. Stat. Mech. (2004) P06002.

[60] M. M. Wolf, F. Verstraete, M. B. Hastings, and J. I. Cirac, Area Laws in Quantum Systems: Mutual Information and Correlations, Phys. Rev. Lett. 100, 070502 (2008). 
[61] M. Tuveri and M. Cadoni, Galactic dynamics and longrange quantum gravity, Phys. Rev. D 100, 024029 (2019).

[62] M. Cadoni, M. Tuveri, and A. P. Sanna, Long-range quantum gravity, Symmetry 12, 1396 (2020).

[63] R. Brustein, A. J. M. Medved, and K. Yagi, Lower limit on the entropy of black holes as inferred from gravitational wave observations, Phys. Rev. D 100, 104009 (2019).

[64] G. Carullo, D. Laghi, J. Veitch, and W. Del Pozzo, Bekenstein-Hod Universal Bound on Information Emission Rate Is Obeyed by LIGO-Virgo Binary Black Hole Remnants, Phys. Rev. Lett. 126, 161102 (2021).
[65] W. G. Unruh, Experimental Black Hole Evaporation, Phys. Rev. Lett. 46, 1351 (1981).

[66] A. A. Svidzinsky, Excitation of a uniformly moving atom through vacuum fluctuations, Phys. Rev. Research 1, 033027 (2019).

[67] M. Mannarelli, D. Grasso, S. Trabucco, and M. L. Chiofalo, Hawking temperature and phonon emission in acoustic holes, Phys. Rev. D 103, 076001 (2021).

[68] C. Barcelo, S. Liberati, and M. Visser, Analogue gravity, Living Rev. Relativity 8, 12 (2005). 